\title{
Effect of clustering in a complex user network on the telephone traffic
}

\author{
Wai M. Tam ${ }^{\mathrm{a}, *}$, Francis C.M. Lau ${ }^{\mathrm{a}}$, Chi K. Tse ${ }^{\mathrm{a}}$, Yongxiang Xia ${ }^{\mathrm{a}}$, Xiuming Shan ${ }^{\mathrm{b}}$ \\ ${ }^{a}$ Department of Electronic and Information Engineering, The Hong Kong Polytechnic University, Hong Kong, China \\ ${ }^{\mathrm{b}}$ Department of Electronic Engineering, Tsinghua University, Beijing, China
}

Received 21 January 2006; received in revised form 30 March 2006

Available online 2 May 2006

\begin{abstract}
Recently, it has been found that the degree distribution of a user network has a significant effect on the traffic carried by a telephone network. In this paper, we investigate the effect of user clustering on the telephone traffic based on computer simulations. Both uniform and scale-free user networks have been considered. In a uniform user network, it has been found that the carried traffic is not affected by the clustering. On the other hand, a scale-free user network with high clustering produces more carried traffic compared to one with smaller clustering because of the larger call arrival rate and the smaller call blocking rate.
\end{abstract}

(C) 2006 Elsevier B.V. All rights reserved.

Keywords: Clustering; Scale-free; User network; Telephone traffic

\section{Introduction}

Studies of complex networks have shown that the amount of clustering among the nodes can affect the performance of the networks. For example, when simple particle hopping models are used for modeling traffic flow in a communication network, results show that the dynamics is slowed down with a high clustering [1]. In another study, the computational performance of the Hopfield neural network model is found to improve with lower clustering for the same network structure [2].

In the human society, networks formed by people are usually highly clustered $[3,4]$. For example, colleagues working in the same organization are normally familiar with one another, thus forming a cluster. Also, two strangers become friends when they are introduced by a common friend, and the clustering of human networks is thus increased. In addition, studies have shown that the degree of many real networks follows a power-law distribution [5-7]. Such networks are also referred to as scale-free networks. By incorporating clustering into scale-free networks, a class of clustered, scale-free networks are formed and they have been widely investigated [8-12].

\footnotetext{
*Corresponding author. Tel.: + 85227666252 ; fax: + 85223628439

E-mail address: tamwm@eie.polyu.edu.hk (W.M. Tam).
} 
The early application of the power-law characteristic to the study of telephone calls can be found in Ref. [13]. Subsequently, the power-law distribution has been used to describe the number of acquaintances in the user network for the study of telephone traffic [14]. In Ref. [14], it is assumed that each user makes calls to his acquaintances. Also, users with a large number of acquaintances will make/receive more calls, whereas users with a small number of acquaintances will have fewer calls.

In this paper, we consider uniform and scale-free user networks. By varying the degree distribution and clustering of the user networks, we study the effect on the traffic carried in a telephone network.

\section{User network model}

Consider a network with $N$ users. The users are represented by nodes in a graph whereas a connection between any two nodes indicates that the two corresponding users are acquaintances (friends or relatives) of each other. For a uniform user network, each node will have the same degree. For a scale-free user network, the degree distribution of the nodes follows a power law given by $P(k) \sim k^{-\gamma}$, where $P(k)$ is the probability of a node with degree $k$ and $\gamma$ is the scaling exponent.

Scale-free networks can be created based on the Barabási-Albert (BA) or modified BA models $[6,15,16]$. In our study, the modified BA model will be used. We start with a network with $m$ isolated nodes. At each time step, a new node together with $m$ outgoing links is added to the network. The probability for each new link to connect to node $i$ will be proportional to $k_{i}+k_{0}$, where $k_{i}$ represents the degree of node $i$ and $-m<k_{0}<\infty$. Also, the exponent for the resultant network has been shown equal to $\gamma=$ $3+k_{0} / m[15,16]$.

After generating a uniform or scale-free user network, we alter the clustering of the network based on the technique described as follows. We first record the original degree of each of the nodes and we remove all links in the network. Then we reset the number of established connections of all nodes, denoted by $n_{i}, i=1,2, \ldots, N$, to zero. A value between 0 and 1 is selected for the control parameter $p$. Setting $i=1$, we begin with the first node and reconnect the links of the nodes sequentially according to following algorithm.

(1) Check whether all links of node $i$ are established: Compare the number of established connections $n_{i}$ with the original degree of the node $k_{i}$. If $n_{i}=k_{i}$, set $i=i+1$ and repeat Step 1 .

(2) Find a node $j$ which is not connected to node $i$ : Generate a random variable $q$ uniformly distributed between 0 and 1 .

(a) If $q \leqslant p$, choose an integer $j$ randomly from 1 to $N$ with equal probability. If $j=i$ or there is already a link between node $i$ and node $j$, repeat Step 2(a).

(b) If $q>p$, set $j=i+1$.

(c) If there is already a link between node $i$ and node $j$, set $j=j+1$ and repeat Step 2(c).

(3) Check whether all $k_{j}$ links of node $j$ are established: If $n_{j}=k_{j}$, go to Step 2.

(4) Link establishment: Establish a link between node $i$ and node $j$. Set $n_{i}=n_{i}+1$ and $n_{j}=n_{j}+1$.

(5) Check whether the reconnection process of all $N$ nodes has been completed: If $n_{i}=k_{i}, i=1,2, \ldots, N$, exit the reconnection algorithm; otherwise, go to Step 1.

\section{Traffic model}

Depending on whether a user is being involved in a call or not, the user is considered as active or inactive. We also denote the state of the user as either "busy" or "idle". Fig. 1 illustrates the different scenarios when new calls are initiated. For an inactive user initiating a call, the call is either successful or blocked, depending on the callee being "idle" or "busy". (Here, we assume that the telephone system can provide sufficient channels for communication between users such that the calls will not be blocked due to channel limitations.) If an active user attempts to make a call, such calls will be "unrealized" regardless of the state of the callee. It is because we assume that anyone in the "active" state should not make any new calls [14]. 


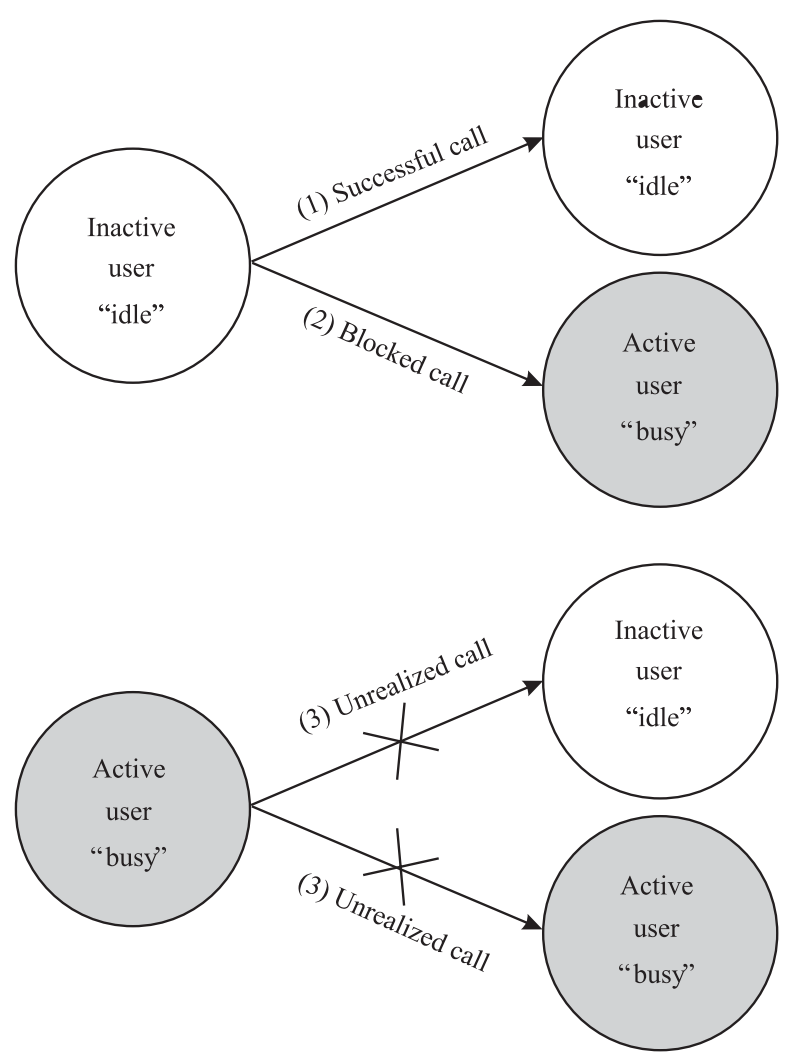

Fig. 1. An active/inactive user is at a busy/idle state, and is/is not engaged in a conversation. Calls initiated by inactive users are either successful or blocked, whereas calls initiated by active users are always "unrealized".

For the $i$ th user, the traffic is generated according to the following model. We assume that the intercall time $t$ follows an exponential distribution, i.e., the probability density function (pdf) of $t$ equals [14]

$$
f(t)=\lambda_{i} \mathrm{e}^{-\lambda_{i} t}
$$

In (1), $\lambda_{i}$ is assumed to be proportional to the number of acquaintances of the $i$ th user, i.e.,

$$
\lambda_{i}=\alpha k_{i}
$$

where $\alpha>0$ is a proportionality constant that will determine the average call arrival rate among all users. For established calls, the call holding time $T_{c}$ has a negative exponential distribution and the pdf is given by

$$
g\left(T_{c}\right)=\mu \mathrm{e}^{-\mu T_{c}},
$$

where $1 / \mu$ denotes the average call duration.

\section{Results and discussions}

We study the telephone traffic based on simulations and we consider $N=10,000$ users. In the construction of the scale-free user network, we use $m=3$ and $k_{0}=-2.7$, giving an average node degree $\bar{k}=$ $(1 / N) \sum_{i=1}^{N} k_{i}=6$ and a scaling exponent $\gamma=2.1$. We also assume $\alpha=1 / 300 \mathrm{call} /(\mathrm{min}$ acquaintance) and $1 / \mu=4 \mathrm{~min}$. Hence the average call arrival rate $\bar{\lambda}$ equals $\alpha \bar{k}=0.02$ call $/ \mathrm{min}$ for the scale-free user network. To provide a fair comparison, we assume that each user is acquainted with six other users and the average call arrival rate for each user equals $0.02 \mathrm{call} / \mathrm{min}$ in the uniform user network. 


\subsection{Uniform user network}

Fig. 2 plots the clustering coefficient $C$ for the uniform user network when we vary the control parameter $p$ in the network construction process. It can be observed that a wide range of clustering coefficient can be obtained by choosing an appropriate value for $p$, and that the clustering coefficient decreases with $p$. In particular, at $p=0$ and $1, C=0.60$ and $4 \times 10^{-4}$, respectively.

Fig. 3 plots the new call arrival rates and new call blocking rates for the uniform user networks. It can be found that the call arrival rates and call blocking rates are not affected by the control parameter $p$, implying that they are independent of the clustering coefficient. As a consequence, the carried traffic is expected to be independent of the value of $p$ or the clustering coefficient, as can be readily observed in Fig. 4.

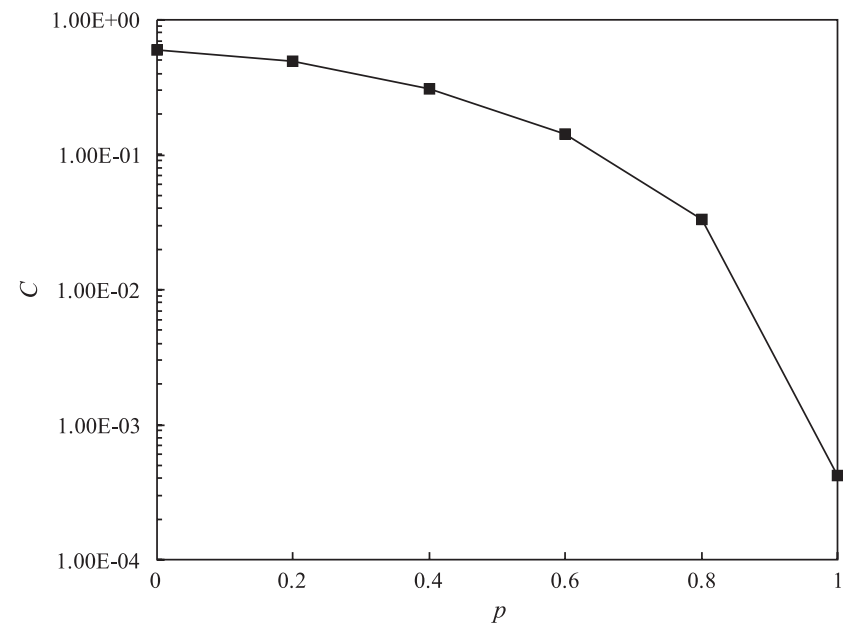

Fig. 2. The clustering coefficient $C$ of the uniform user network versus the control parameter $p$ used in the construction of the network. A user is connected to a randomly selected user with probability $p$, and $p=1$ corresponds to a random network with uniform degree distribution.

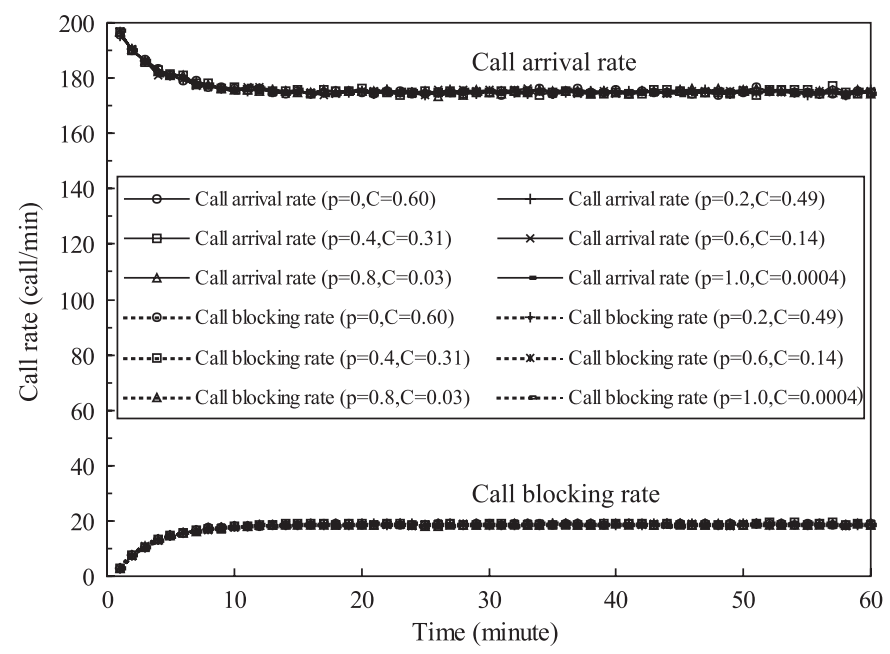

Fig. 3. New call arrival rates and new call blocking rates versus time for the uniform user networks with control parameter $p=0,0.2,0.4$, $0.6,0.8$ and 1.0 (correspondingly, clustering coefficient $C=0.60,0.49,0.31,0.14,0.03$ and 0.0004 ). The same set of user degrees is used for the user network with different clustering coefficients. The clustering coefficient makes no impact on the new call arrival rates and new call blocking rates. 


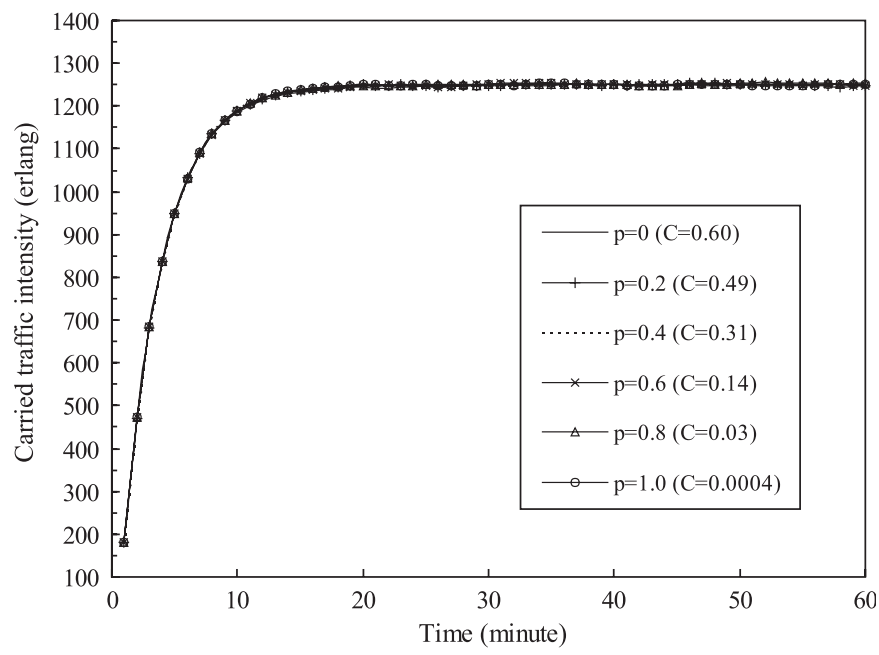

Fig. 4. Carried traffic intensity versus time for the uniform user networks with control parameter $p=0,0.2,0.4,0.6,0.8$ and 1.0 (correspondingly, clustering coefficient $C=0.60,0.49,0.31,0.14,0.03$ and 0.0004 ). The carried traffic is the sum of the call service time of all users for a given time period, i.e., $1 \mathrm{~min}$, which is found to be independent of the clustering coefficient.

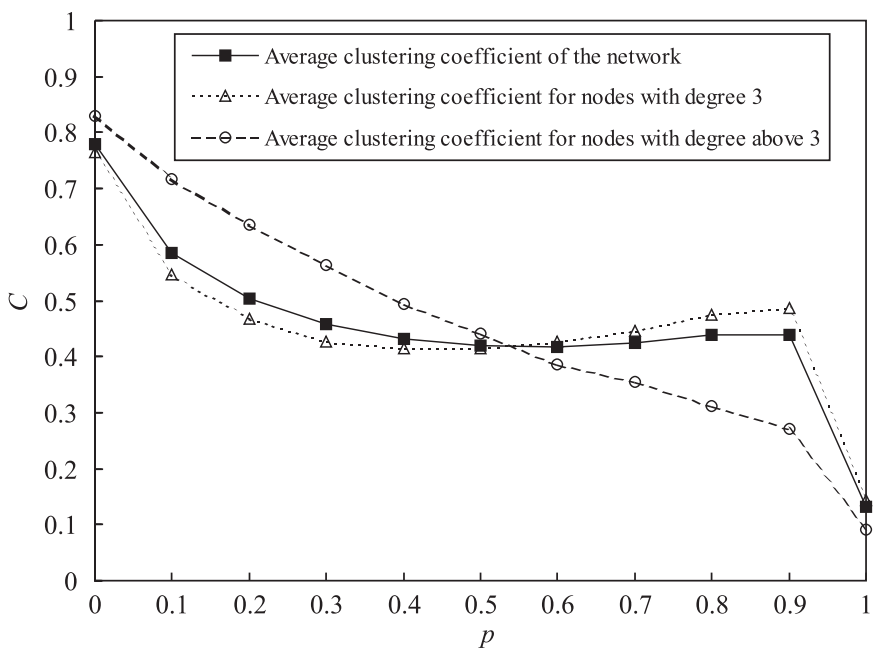

Fig. 5. The clustering coefficient $C$ of the scale-free user network versus the control parameter $p$ used in the construction of the network. A user is connected to a randomly selected user with probability $p$, and $p=1$ corresponds to a random network with scale-free degree distribution. For nodes with degree above 3, the average clustering coefficient decreases steadily with $p$. For nodes with degree equals 3 , the average clustering coefficient decreases initially, then increases slightly before dropping again. The average clustering coefficient of the whole network follows a similar trend as that for nodes with degree equals 3 .

\subsection{Scale-free user network}

Fig. 5 plots the clustering coefficient $C$ of the resultant scale-free user network when we vary the control parameter $p$ during the network construction process. Note that for any individual user, the number of acquaintances (node degree) remains the same even when we vary $p$. Based on the graph, it is found that for nodes with degree above 3 , the average clustering coefficient among these nodes decreases steadily with $p$. But for nodes with degree equals 3 , the average clustering coefficient decreases initially when $p$ is increased from 0.1 to 0.4 . Then the clustering coefficient increases slightly when $p$ is increased from 0.4 to 0.9 , before dropping again when $p$ is increased to 1.0. Because of the large number of nodes with degree 3 , the average clustering 
coefficient of the whole network is dominated by and follows a similar trend as that for nodes with degree equals 3.

Fig. 6 plots the new call arrival rates and new call blocking rates for the scale-free user networks. Results show that in general, the call arrival rate decreases and the call blocking rate increases with $p$. Consequently, the carried traffic decreases with $p$, as shown in Fig. 7. Comparing the results with those in Fig. 5, it appears that for the scale-free user networks, the call arrival rates, the call blocking rates and the carried traffic change monotonically with the average clustering coefficient of nodes with degree more than 3 .

Next, we study in detail the cases in which the control parameter $p$ equals 0 and 1 , respectively, corresponding to a highly clustered scale-free user network and a loosely clustered one. Fig. 8 plots the carried traffic intensity against the average call arrival rate $\bar{\lambda}$, which is adjusted by changing the value of the proportionality constant $\alpha$ in (2). The results show that more traffic can be carried as the average call arrival

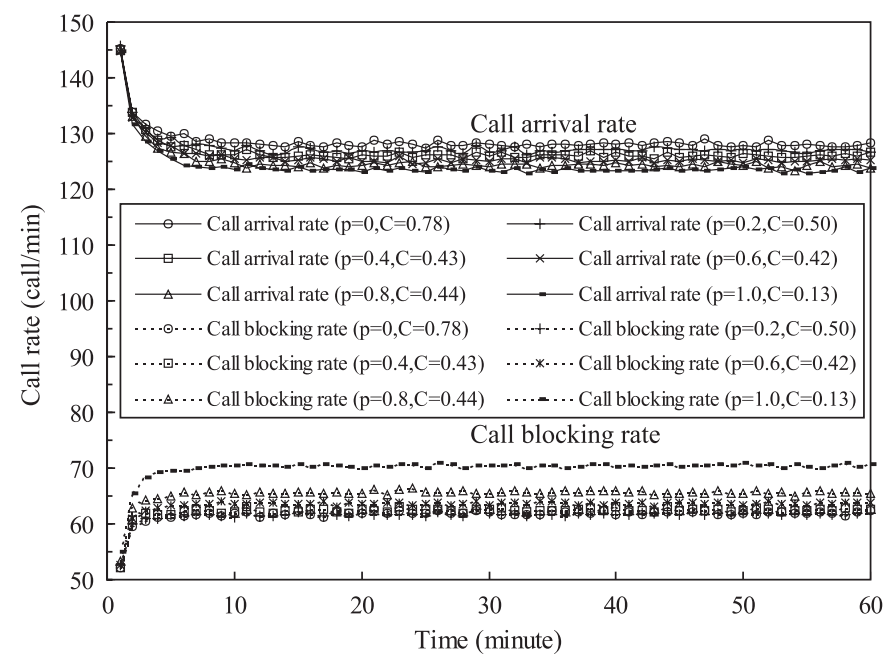

Fig. 6. New call arrival rates and new call blocking rates versus time for the scale-free user networks with control parameter $p=0,0.2$, $0.4,0.6,0.8$ and 1.0 (correspondingly, clustering coefficient $C=0.78,0.50,0.43,0.42,0.44$ and 0.13 ). Call arrival rate decreases and call blocking rate increases with $p$.

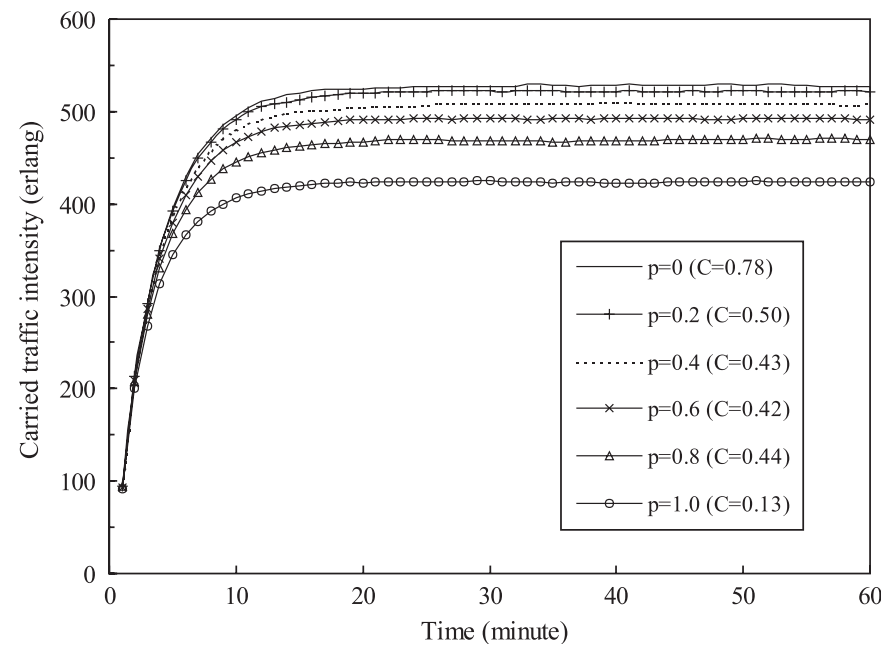

Fig. 7. Carried traffic intensity versus time for the scale-free user networks with control parameter $p=0,0.2,0.4,0.6,0.8$ and 1.0 (correspondingly, clustering coefficient $C=0.78,0.50,0.43,0.42,0.44$ and 0.13 ). The carried traffic decreases with $p$. 


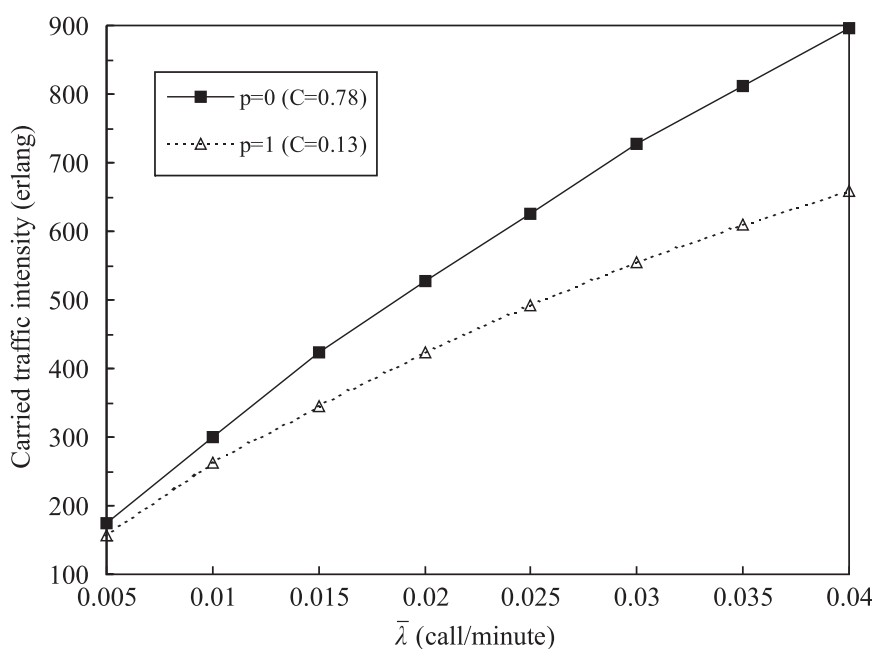

Fig. 8. Carried traffic intensity versus average call arrival rate $\bar{\lambda} . \gamma=2.1$ and $1 / \mu=4 \mathrm{~min}$. The carried traffic intensity increases with average call arrival rate. A highly clustered user network ( $p=0$ or $C=0.78)$ produces more carried traffic compared with a loosely clustered user network ( $p=1$ or $C=0.13)$.

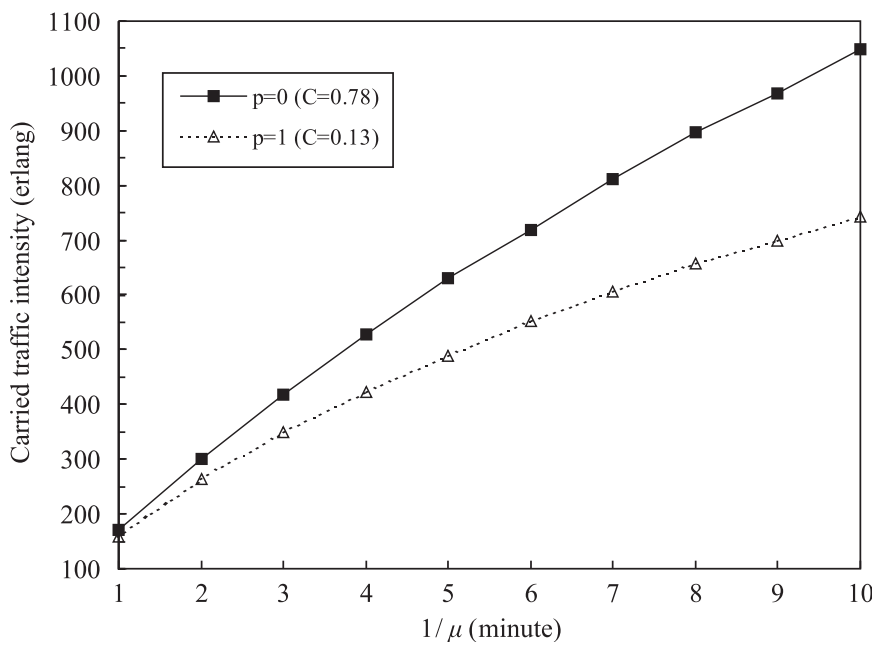

Fig. 9. Carried traffic intensity versus average call duration $1 / \mu . \gamma=2.1$ and $\alpha=1 / 300$ call/(min acquaintance). The carried traffic intensity increases with average call duration. A highly clustered user network $(p=0$ or $C=0.78)$ produces more carried traffic compared with a loosely clustered user network $(p=1$ or $C=0.13)$.

rate and hence offered traffic increases. In Fig. 9, we investigate the effect of the average call duration $1 / \mu$ on the carried traffic. As the average call duration increases, more traffic is being offered and it is found that more traffic is carried by the network. Based on the results in Figs. 8 and 9, it can be observed that there is relatively more carried traffic in a highly clustered scale-free user network $(p=0)$ compared to a loosely clustered one $(p=1)$. Moreover, the discrepancy in carried traffic widens as the amount of offered traffic increases.

Finally, we investigate the effect of the scaling exponent $\gamma$ on the carried traffic. A smaller value for $\gamma$ implies that there is a relatively large number of users with a relatively large number of acquaintances. In this study, for each value of $\gamma$, a new scale-free network needs to be created based on the modified BA method. Then the links are reconnected for the cases $p=0$ and 1 , respectively. Therefore, when $\gamma$ varies, both the degree distribution and the clustering of the network change. However, the fact that the clustering coefficient 


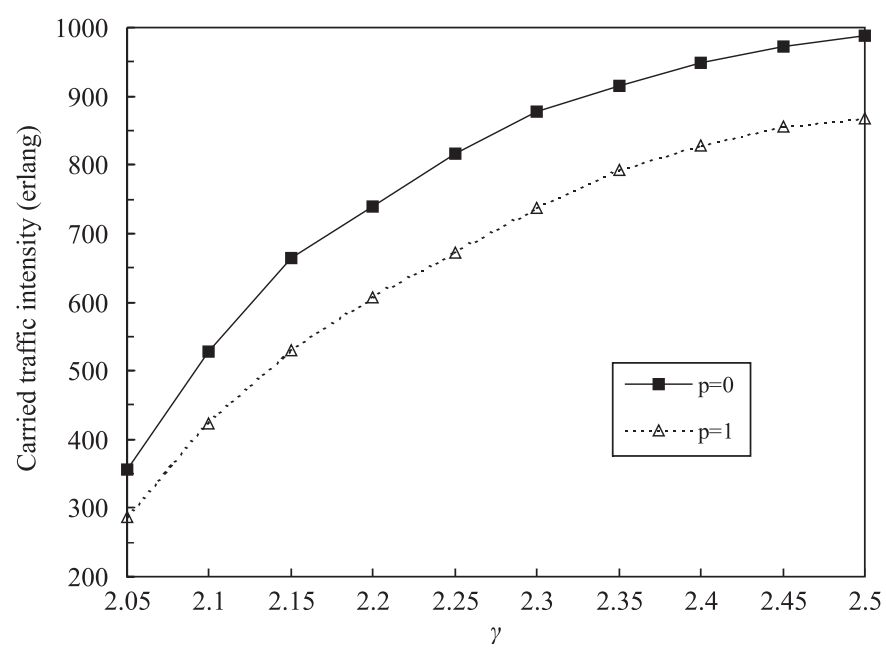

Fig. 10. Average carried traffic intensity versus scaling exponent $\gamma . \alpha=1 / 300$ call $/(\min$ acquaintance $)$ and $\bar{\lambda}=0.02$ call $/$ min. A smaller scaling exponent implies that there is a relatively large number of users with a relatively large number of acquaintances. Both the degree distribution and the clustering of the network change with the scaling exponent. The clustering coefficient corresponding to $p=0$ is always larger than that of $p=1$. The carried traffic intensity increases with the scaling exponent and that a larger clustering results a higher carried traffic.

corresponding to $p=0$ is larger than that of $p=1$ remains valid. In Fig. 10, we observe that the carried traffic intensity increases with the scaling exponent $\gamma$ and that a larger clustering results a higher carried traffic.

\section{Conclusions}

In this paper, the clustering effect of the user network on the telephone traffic has been studied. Two types of user networks, namely uniform user network and scale-free user network, have been considered. Results show that the clustering has no effect on the traffic of a uniform user network. For a scale-free user network, a larger scaling exponent or a larger clustering in general allows more traffic to be carried by the telephone network.

\section{Acknowledgment}

This work was supported by Hong Kong Polytechnic University under a Postdoctoral Fellowship Grant (number G-YX33).

\section{References}

[1] P. Holme, Congestion and centrality in traffic flow on complex networks, Adv. Complex Systems 6 (2) (2003) $163-176$.

[2] B.J. Kim, Performance of networks of artificial neurons: the role of clustering, Phys. Rev. E 69 (2004) 045101(R).

[3] D.J. Watts, S.H. Strogatz, Collective dynamics of 'small-world' networks, Nature (London) 393 (1998) $440-442$.

[4] M.E.J. Newman, J. Park, Why social networks are different from other types of networks, Phys. Rev. E 68 (2003) 036122.

[5] A.-L. Barabási, R. Albert, Emergence of scaling in random network, Science 286 (1999) 509-512.

[6] R. Albert, A.-L. Barabási, Statistical mechanics of complex networks, Rev. Mod. Phys. 74 (2002) 47-97.

[7] X.F. Wang, G. Chen, Complex networks: small-world, scale-free and beyond, IEEE Circuits \& Systems Mag. 3 (2003) 6-20.

[8] S.N. Dorogovtsev, J.F.F. Mendes, A.N. Samukhin, Size-dependent degree distribution of a scale-free growing network, Phys. Rev. E 63 (2001) 062101.

[9] P. Holme, B.J. Kim, Growing scale-free networks with tunable clustering, Phys. Rev. E 65 (2002) 026107.

[10] H. Kim, Y. Choi, J.M. Kim, Stochastic evolving model for complex networks, Mod. Phys. Lett. B 18 (23) (2004) $1157-1164$.

[11] K. Klemm, V.M. Eguíluz, Highly clustered scale-free networks, Phys. Rev. E 65 (2002) 036123.

[12] K. Klemm, V.M. Eguíluz, Growing scale-free networks with small-world behavior, Phys. Rev. E 65 (2002) 057102. 
[13] W. Aiello, F. Chung, L. Lu, A random graph model for massive graphs, Proceedings of 32nd Annual ACM Symposium on Theory of Computing, ACM, New York, 2000, pp. 171-180.

[14] Y.X. Xia, C.K. Tse, W.M. Tam, F.C.M. Lau, M. Small, Scale-free user network approach to telephone network traffic analysis, Phys. Rev. E 72 (2005) 026116.

[15] S.N. Dorogovtsev, J.F.F. Mendes, A.N. Samukhin, Structure of growing networks with preferential linking, Phys. Rev. Lett. 85 (21) (2000) 4633-4636.

[16] X. Yao, C. Zhang, J. Chen, Y. Li, On the formation of degree and cluster-degree correlations in scale-free networks, Physica A 353 (2005) 661-673. 